Research Article

\title{
The Role of Adenosine A2b Receptor in Mediating the Cardioprotection of Electroacupuncture Pretreatment via Influencing $\mathrm{Ca}^{2+}$ Key Regulators
}

\author{
Qiu-Fu Dai $\mathbb{D}^{1},{ }^{1}$ Jun-Hong Gao, ${ }^{2}$ Juan-Juan Xin, ${ }^{2}$ Qun Liu, ${ }^{2}$ Xiang-Hong Jing $\mathbb{D}^{2}$ \\ and Xiao-Chun Yu $\mathbb{D}^{2}$ \\ ${ }^{1}$ Acupuncture and Moxibustion Department, Beijing Hospital of Traditional Chinese Medicine Affiliated to Capital \\ Medical University, Capital Medical University, Beijing Key Laboratory of Acupuncture Neuromodulation, \\ 23 Art Museum Back Street, Beijing 100700, China \\ ${ }^{2}$ Institute of Acupuncture and Moxibustion, China Academy of Chinese Medical Sciences, 16 Nanxiaojie, Dongzhimennei, \\ Beijing 100700, China \\ Correspondence should be addressed to Xiao-Chun Yu; yuxc@mail.cintcm.ac.cn
}

Received 25 July 2019; Revised 29 October 2019; Accepted 11 November 2019; Published 2 December 2019

Academic Editor: Giacinto Bagetta

Copyright (c) 2019 Qiu-Fu Dai et al. This is an open access article distributed under the Creative Commons Attribution License, which permits unrestricted use, distribution, and reproduction in any medium, provided the original work is properly cited.

\begin{abstract}
Objective. To investigate the roles played by $\mathrm{A} 2 \mathrm{~b}$ receptor and the key $\mathrm{Ca}^{2+}$ signaling components in the mediation of the cardioprotection of electroacupuncture pretreatment in the rats subjected to myocardial ischemia and reperfusion. Methods. SD rats were randomly divided into a normal control (NC) group, ischemia/reperfusion model (M) group, electroacupuncture pretreatment (EA) group, and electroacupuncture pretreatment plus A2b antagonist (EAG) group. The ischemia/reperfusion model was made by ligation and loosening of the left descending branch of the coronary artery in all groups except the NC group. The EA group was pretreated with electroacupuncture at the Neiguan (PC6) point once a day for three consecutive days before the modeling. The elevation of the ST segment, arrhythmia scores, and myocardial infarction size of each group was measured. The relative expression levels of A2b, RyR2, SERCA2a, NCX1, P-PLB(S16)/PLB, and Troponin C/Troponin I proteins in the injured myocardium were detected by multiple fluorescence western blot. Results. The level of ST segment, arrhythmia scores, and infarct size in the M group was significantly higher/larger than that in the NC group after ischemia and reperfusion, while all the three indices mentioned above in the EA group were significantly lower/smaller than those in the M group after reperfusion. The expression of the proteins of adenosine receptor $2 \mathrm{~b}(\mathrm{~A} 2 \mathrm{~b})$, ryanodine receptor 2(RyR2), and sarco(endo)plasmic reticulum $\mathrm{Ca}^{2+}$ ATPase 2a (SERCA2a) in the EA group was significantly enhanced as compared with the M group, while in the EAG group, the contents of A2b were significantly lower than those in the EA group, and RyR2 was higher in the EAG group. In comparison with the NC group, the relative expression of NCX1 protein in M, EA, and EAG groups was not changed significantly. The ratio of phosphorylated phospholamban (P-PLB) over phospholamban (PLB) in the M group was significantly lower than that in the NC group, and the ratio in the EA group was significantly increased as compared with the M group, while the ratio of Troponin C/ Troponin I in the EA group was significantly decreased in comparison with that in other groups. Conclusion. Electroacupuncture pretreatment could reduce ischemia and reperfusion-induced myocardial injury via possibly increasing the A2b content and regulating the key $\mathrm{Ca}^{2+}$ signaling components, namely inhibiting RyR2 and enhancing P-PLB(S16)/PLB ratio and SERCA2a proteins, so as to diminish the intracellular $\mathrm{Ca}^{2+}$ overload and consequently lessen the myocardial injury.
\end{abstract}

\section{Introduction}

Ischemic heart disease (IHD) is one of the diseases with the highest morbidity and mortality over the world. In China, there were about 4 million patients attacked by the coronary heart disease in 2016 [1]. The studies showed that in patients with IHD, a further myocardial injury can be caused by the ischemia/reperfusion(I/R) $[2,3]$. In the recent decades, it has been a hot topic to find out a safe and effective approach to the prevention and treatment of the reperfusion-induced 
myocardial injury. Ely and his colleagues reported [4] previously that adenosine released during myocardial ischemia produced a direct cardioprotection. Adenosine receptors were reported to mediate not only the cardioprotection induced by ischemic preconditioning $[5,6]$ but also the inhibition of the apoptosis of cardiac cells during the reperfusion [7]. Among the well-known 4 adenosine receptors, the subtype adenosine receptor $2 \mathrm{~b}(\mathrm{~A} 2 \mathrm{~b})$ was proved to mediate the cardioprotective effects induced by both ischemic preconditioning and postconditioning [8]. In the rats with $\mathrm{A} 2 \mathrm{~b}$ gene knocked out, there were not any cardioprotective effects observed, while the ischemic preconditioning could still produce the cardioprotection in rats with A1, A2a, or A3 gene knocked out [9]. The results indicated that $\mathrm{A} 2 \mathrm{~b}$ receptor played an important role in the mediation of the cardioprotection. It was showed in the clinical studies that the myocardial injury was reduced effectively by the acupuncture pretreatment in patients with myocardial ischemia $[10,11]$. The results achieved by a lot of experimental studies indicated that the incidence rates of sudden death [12], arrhythmias, and angina pectoris were significantly diminished by acupuncture [13, 14]. Acupuncture stimulation was also showed to alter both the local adenosine concentration in the tissues around the acupoints [15] and the expression of A2b receptor in cardiac cells [16]. Accordingly, it is highly likely that A2b participates in the cardioprotection produced by acupuncture pretreatment. It is well known that intracellular calcium overload contributes to the myocardial ischemic injury, and $\mathrm{A} 2 \mathrm{~b}$ is involved in the modulation of the intracellular calcium concentration [17-19]. The aim of present study is to investigate the role played by $\mathrm{A} 2 \mathrm{~b}$ receptor and the key $\mathrm{Ca}^{2+}$ signaling components in the mediation of the cardioprotection produced by acupuncture pretreatment. The outcomes will provide the scientific evidence to support acupuncture as an applicable way to effectively prevent and control the IHD.

\section{Materials and Methods}

2.1. Animals. Forty-eight male Sprague Dawley (SD) rats weighing $300 \pm 25 \mathrm{~g}$ were purchased from Experimental Animal Central of Peking Union Medical University (Certifcate number SCX2016-0002, Beijing, China). The rats were kept in an animal house maintained at $21 \pm 2^{\circ} \mathrm{C}$ with a 12-hour light-dark cycle and freed to have food and water. All experiments conducted in the present studies involving animals were in accordance with the ethical standards of the Institutional Animal Care and Use Committee of the China Academy of Chinese Medical Sciences.

2.2. Grouping and Pretreatment. The rats were acclimatized for a week, and randomized into a Normal control (NC) group, Model (M) group, Electroacupuncture pretreatment (EA) group, and Electroacupuncture pretreatment plus A2b antagonist (EAG) group, with 12 rats in each group. The experimental protocol was described in Figure 1. The NC group was only punctured under the left descending branch of coronary artery (LCA) without ligation and electroacupuncture pretreatment. The root of LCA was ligated in the $\mathrm{M}$ group without electroacupuncture pretreatment, and the rats in the $\mathrm{NC}$ group received threading but not ligation. The EA group was pretreated with electroacupuncture pretreatment applied at bilateral Neiguan (PC6) acupoints for $30 \mathrm{~min}$ once a day for 3 consecutive days. The acupuncture needle was $0.3 \times 25 \mathrm{~mm}$ (Huatuo, China), and needling depth was about $2 \mathrm{~mm}$. An A2b antagonist GS6201 (Tocris Bioscience, No.4727) was administered intraperitoneally in the EAG group at a dose of $1 \mathrm{mg} /$ $\mathrm{kg}, 2 \mathrm{~h}$ before EA pretreatment twice a day for 3 consecutive days [20]. The acupoints were located in the forelimbs according to the textbook of experimental acupuncture in animals and stimulated with an intensity of $1 \mathrm{~mA}$ and a frequency of $2 / 10 \mathrm{~Hz}$ in the present study.

\subsection{Acute Myocardial Ischemia and Reperfusion Model.} The rats were anesthetized by intraperitoneal injection of $20 \%$ urethane $(0.5 \mathrm{ml} / 100 \mathrm{~g})$ and placed on a temperaturecontrolled heated table to maintain body temperature at $37^{\circ} \mathrm{C}$. The trachea was intubated for artificial respiration. After a recovery of $20 \mathrm{~min}$ following the thoracotomy, LCA was ligated for $30 \mathrm{~min}$, and the model was established according to our previous research [21]. Successful LCA occlusion was confirmed by an immediate color change of the myocardium below the ligation site from red to dark violet, as well as the immediate occurrence of ST elevations in the electrocardiogram (ECG). After 30-min ischemia, the rats received reperfusion for $15 \mathrm{~min}$ among the $\mathrm{M}, \mathrm{EA}$, and EAG groups.

\subsection{Observation and Detection Methods}

(1) ST segment changes in ECG: the ST segment is defined as $13 \mathrm{~ms}$ after the $\mathrm{S}$ wave [22]. The ST segment elevation was measured before coronary ligation and $15 \mathrm{~min}$ and $30 \mathrm{~min}$ after ligation, as well as $15 \mathrm{~min}$ after reperfusion, respectively.

(2) Arrhythmia scoring system: Curtis and Walker (1988) arrhythmia scoring method was used [23, 24]; the arrhythmias of each group were scored within $15 \mathrm{~min}$ after reperfusion, and the details of the scoring system are as follows (Table 1).

(3) Determination of ischemic risk zone and infarct size.

The ischemic risk zone and infarct size were determined by Evans blue-TTC double staining. Experimental methods refer to reference [25]; the precooling saline was used to wash off redundant dye from the removed heart, excess water was blotted up by using a filter paper, and the heart was frozen to $-20^{\circ} \mathrm{C}$ for $15 \mathrm{~min}$. The frozen myocardium was cut into 5 thin slices with a thickness of $2 \mathrm{~mm}$ along the ligation position. The digital camera was used to take pictures. Image-Pro plus 6.0 software was used to calculate the infarct size and risk zone. After staining, the myocardial tissue showed blue as normal tissue, red as risk zone, and pale as infarct size. The ratio of infarct size/risk zone represents the extent of the infarct. 


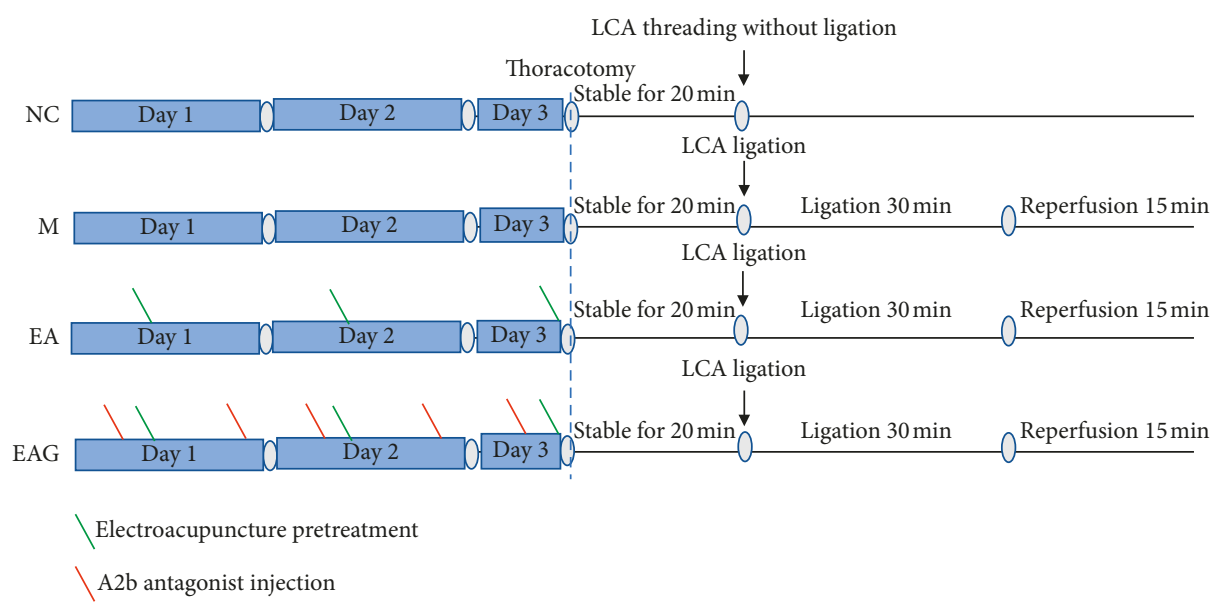

FIGURE 1: The experimental protocol.

Table 1: Arrhythmia scoring system.

\begin{tabular}{lc}
\hline Arrhythmia scores & Type of arrhythmia \\
\hline 0 & No arrhythmia \\
1 & Atrial arrhythmias or an \\
2 & occasional PVC \\
3 & Frequent PVC \\
4 & VT (1 2 episodes) \\
\hline
\end{tabular}

PVC, premature ventricular contraction; VT, ventricular tachycardia; VF, ventricular fibrillation.

2.5. Multiple Fluorescence Western Blot. Cardiac tissue was cracked by RIPA and phosphatase inhibitor (Bimake, B15001) mixture to extract protein. Protein concentration was determined by BCA protein reagents (Thermo scientific, no. 23227). $20 \mu \mathrm{g}$ of proteins were resolved on SDS-PAGE, transferred to a low-fluorescence PVDF membrane (Thermo scientific, no. 22860), and incubated with the following primary antibodies: anti-A2b (1:1000, GeneTex54903), antiphospholamban (PLB, 1:5000, Abcam2865), antiphospholamban phosphor S16 (P-PLB, $0.5 \mu \mathrm{g} / \mathrm{ml}$, Abcam 15000), antiryanodine receptor 2 (RyR 2, 1:5000, Abcam2861), NCX1 (1:1000, Abcam177952), SERCA2a (1: 1000, Abcam2861), troponin C (1:4000, Abcam137130), and troponin I $(1: 2000$, Abcam10231). All of the membranes were incubated at $4^{\circ} \mathrm{C}$ overnight. After incubation with fluorescent secondary antibodies (1:15000, Licor, IRDye $\left.{ }^{\circledR} 800 \mathrm{CW}, \mathrm{IRDye}^{\circledR} 680 \mathrm{RD}\right)$ for $1 \mathrm{~h}$ at room temperature, the membrane was washed 3 times and incubated with the housekeeping protein $\mathrm{HFAB}^{\mathrm{TM}}$ Rhodamine anti-GAPDH $(1: 1000$, Bio-rad 12004167) for $1 \mathrm{~h}$ at room temperature. Images were taken with a Typhoon FLA9500 (GE Healthcare) and analyzed by using ImageQuant TL software.

2.6. Statistical Analysis. All data are expressed as mean\pm SEM. SPSS13.0 software was used for statistical analysis. Normality and equality of variance were tested by the Shapiro-Wilk and Levene test, respectively. Significant difference between groups was determined by one-way ANOVA followed by the Bonferroni post hoc test.
Arrhythmia scoring was tested by the Kruskal-Wallis H test. $P<0.05$ was considered as statistically significant.

\section{Results}

3.1. The Effects of Electroacupuncture Pretreatment on ECG ST Segment. There was no statistically significant difference in the ST segment among the NC, M, EA, and EAG group before ligation $(P>0.05$, respectively, Figure 2$)$. The ST segment in the M, EA, and EAG group was significantly increased after ligation and reperfusion as compared with that in the NC group $(P<0.01$ respectively). The results indicate that the abnormal elevation of the ST segment of ECG was caused by myocardial ischemia and reperfusion. However, the elevation of the ST segment in the EA group was significantly lower than that in the $M$ group at the same time points as mentioned above during ligation for $30 \mathrm{~min}$ and reperfusion $(P<0.05, P<0.01)$. The results show that electroacupuncture pretreatment can significantly inhibit the abnormal elevation of the ST segment caused by myocardial I/R injury. Interestingly, the elevation of the ST segment in the EAG group was obviously higher than that in the EA group, indicating that the inhibitory effect of EA pretreatment on the elevated ST segment was reversed by the pretreatment with the specific A2b antagonist.

3.2. Arrhythmia Scoring of Different Groups. As compared with the NC group, arrhythmia scores in the M group was significantly increased (all $P<0.01$, Figure 3 ), while the score in the EA group was significantly decreased $(P<0.01)$. In the EAG group, the score was significantly increased as compared with the EA group $(P<0.05)$.

3.3. Risk Zone/Infarct Size in Different Groups. In comparison with the NC group, the infarct size in the M group was obvious, showing that the myocardial injury was caused successfully by ischemia and reperfusion (Figure 4). The infarct size in the EA group was significantly smaller than that in the M group, which indicates that electroacupuncture 


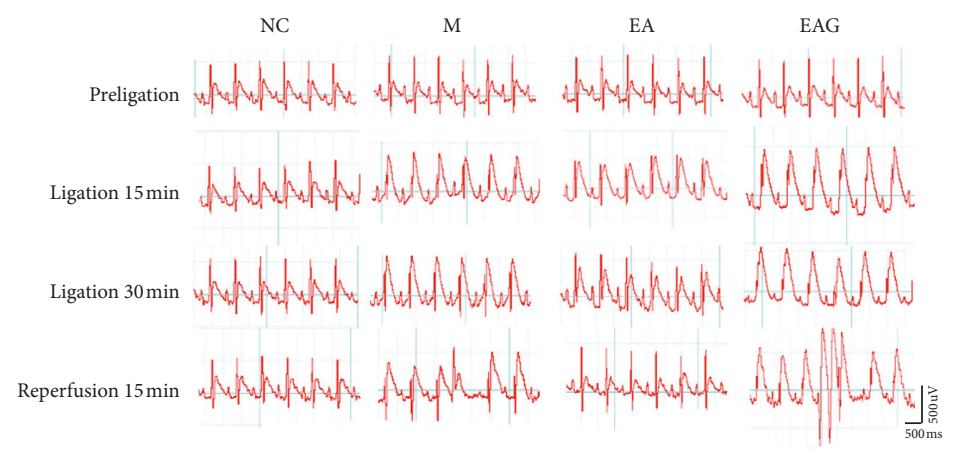

(a)

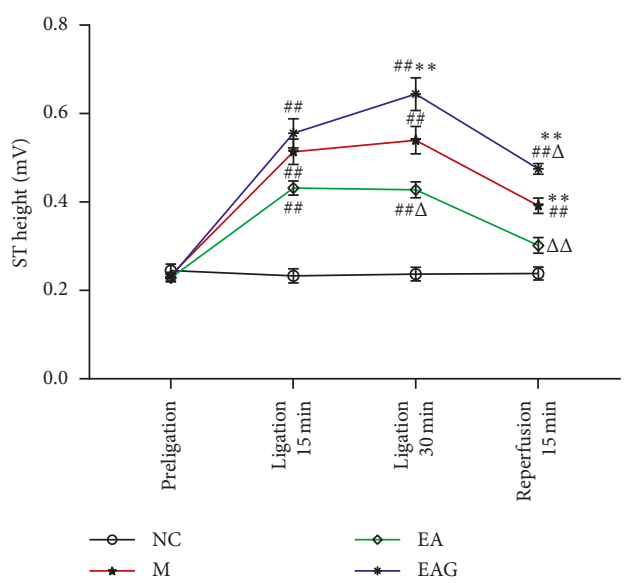

(b)

FIGURE 2: The ECG and ST segments in different groups. $(\mathrm{NC}=$ normal control group, $\mathrm{M}=$ model group, EA=electroacupuncture pretreatment group, and $\mathrm{EAG}=$ electroacupuncture pretreatment plus $\mathrm{A} 2 \mathrm{~b}$ antagonist group. ${ }^{\# \#} P<0.01$, ${ }^{\#} P<0.05$, vs. NC group; ${ }^{\Delta \Delta} P<0.01,{ }^{\Delta} P<0.05$, vs. M group; ${ }^{* *} P<0.01,{ }^{*} P<0.05$, vs. EA group, $n=12$ in each group, $\left.\bar{\chi} \pm S E\right)$.

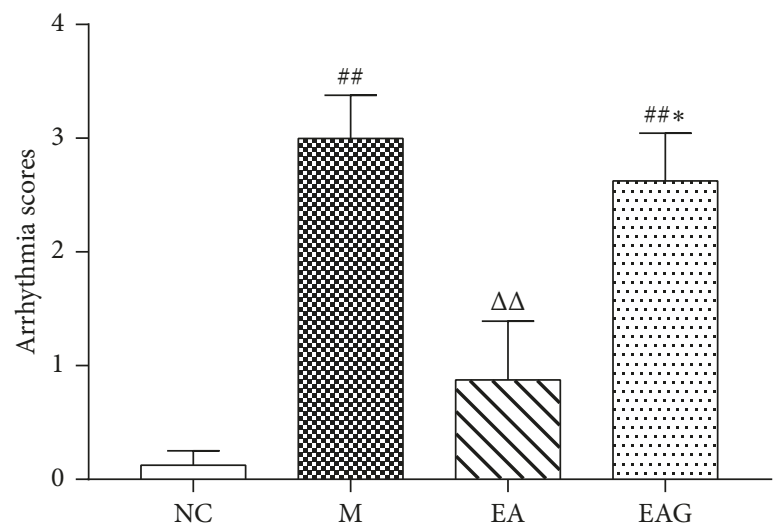

Figure 3: Arrhythmia scoring of different groups. $\left({ }^{\# \#} P<0.01,{ }^{\#} P<0.05\right.$, vs. NC group; ${ }^{\Delta \Delta} P<0.01,{ }^{\Delta} P<0.05$, vs. M group; ${ }^{* *} P<0.01$, ${ }^{*} P<0.05$, vs. EA group, $n=8$ in each group, $\bar{\chi} \pm S E$ ).

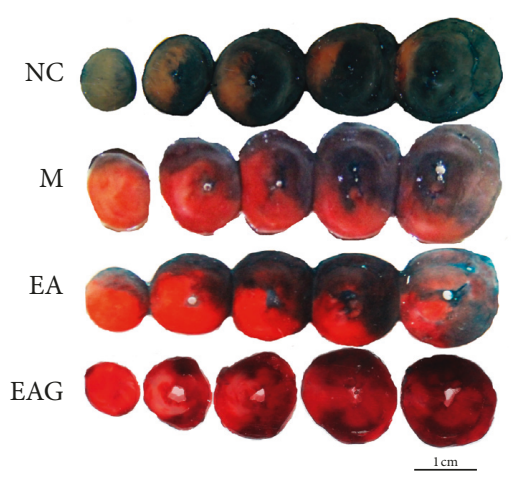

(a)

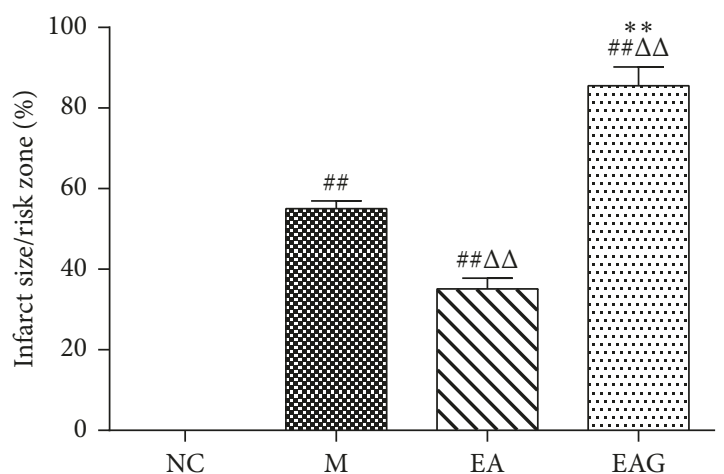

(b)

Figure 4: The typical photos of Evans blue-TTC double staining (a) and the statistical graph showing the myocardial infarct size as percentage of the risk zone in the different groups (b). $\left({ }^{\# \#} P<0.01,{ }^{\#} P<0.05\right.$, vs. NC group; ${ }^{\Delta \Delta} P<0.01,{ }^{\Delta} P<0.05$, vs. M group; ${ }^{* *} P<0.01$, ${ }^{*} P<0.05$, vs. EA group, $n=4$ in each group, $\left.\bar{\chi} \pm S E\right)$.

pretreatment could reduce the myocardial infarction caused by myocardial ischemia. The ratio of infarct size/risk zone in the EAG group was significantly higher than that in the EA group, suggesting that the A2b antagonist (GS6201) can reduce the the cardioprotective effect of electroacupuncture pretreatment. 
3.4. The Relative Expression of the Proteins of $A 2 b, R y R 2$, NCX1, and SERCA2a in the Myocardium. The relative expression of $\mathrm{A} 2 \mathrm{~b}$ in the $\mathrm{M}$ group was significantly higher than that in the NC group $(P<0.01)$. As compared with the $\mathrm{M}$ group, A2b content in the EA group was much higher $(P<0.01)$. In the EAG group, A2b content was significantly lower in comparison with EA group, even lower than that in NC group $(P<0.01)$ (Figure $5(b))$. The relative expression of RyR2 protein in the $M$ group was much higher than that in the NC group. However, as compared with the M group, the content of RyR2 in the EA group was significantly lower $(P<0.01)$. In the EAG group, the content of RyR2 protein was significantly higher than that in the EA group $(P<0.01)$ (Figure 5(c)). As compared with the NC group, the relative expression of NCX1 in the M, EA, and EAG group did not change significantly $(P>0.05)$ (Figure 5(d)). SERCA2a content in the $\mathrm{M}$ group was significantly lower than that in the NC group $(P<0.01)$, while as compared with the $\mathrm{M}$ group, the protein content in the EA group was significantly higher $(P<0.05)$. In the EAG group, it was reduced a little bit but not significantly in comparison with the EA group (Figure 5(e)).

\subsection{The Ratio of P-PLB(S16)/PLB and Troponin C/Troponin I.} The P-PLB/PLB ratio of the $M$ and EAG group was significantly lower than that of the NC group $(P<0.01)$, while the ratio of the EA group was higher than that of the NC and $\mathrm{M}$ group $(P<0.01)$. The ratio of the EAG group was significantly lower than that of the EA group $(P<0.01)$ (Figure 6).

The troponin $\mathrm{C} /$ troponin I ratio of the $\mathrm{M}$ and $\mathrm{EAG}$ group was significantly higher than that of the NC group $(P<0.01)$, while the ratio of the EA group was significantly lower than that of the NC and M group $(P<0.01)$. The ratio of the EAG group was significantly lower than that of the EA group $(P<0.01)$ (Figure 7).

\section{Discussion}

The interesting findings in the present study were that the acupuncture pretreatment could reduce the elevated ECG ST segments, cardiac arrhythmias, and myocardial infarct size significantly in the rats subjected to the myocardial ischemia and reperfusion via enhancing the content of $\mathrm{A} 2 \mathrm{~b}$ receptor and regulating the expression of the key calcium signaling components including RyR2, SERCA2a, and P-PLB(S16)/PLB and subsequently impact the ratio of Troponin C/Troponin I in cardiac muscle (Table 2).

Previous studies have shown that EA pretreatment could prevent myocardial infarction injury by regulating the AMPK/PGC-1 a signaling pathway and AMPK-dependent autophagy process [26, 27], and more and more researchers concern about the acupuncture effects on adenosine receptors. A2b is known to be the major adenosine receptor contributing to the cardioprotection induced by both ischemic preconditioning and postconditioning [28]. Another way for it to mediate the reduction of the ischemia-reperfusion-induced myocardial injury is that activation of the receptor could directly relax the blood vessel and facilitate the regeneration of both blood vessels and cardiomyocytes $[29,30]$. It was reported that acupuncture stimulation could not only change the adenosine concentration around the acupoints locally $[15,31]$ but also regulate the A2b expression in the cardiomyocytes [16]. The present study showed that in the EA group, the electroacupuncture pretreatment significantly enhanced $\mathrm{A} 2 \mathrm{~b}$ content and reduced the elevation of ECG ST segment, arrhythmias, and the myocardial infarct size. The enhancement of $\mathrm{A} 2 \mathrm{~b}$ and the cardioprotective effects were both blocked by the A2bspecific antagonist. In agreement with the previous study, the present results indicate that the cardioprotection produced by electroacupuncture pretreatment is mediated by A2b receptor [32].

Physiologically, calcium is known to be crucial for the systolic and diastolic activities of cardiomyocytes. The disorder of the intracellular calcium concentration like calcium overload is also one of the very important factors leading to pathological alterations of the cardiac cells $[33,34]$. On the calcium signaling pathway of the cardiomyocytes RyR2, NCX, SERCA, and PLB are the key factors or $\mathrm{Ca}^{2+}$ signaling components which play a pivotal role in the balance of the intracellular $\mathrm{Ca}^{2+}$ concentration. For example, RyR2 is the major receptor on the membrane of the sarcoplasmic reticulum responsible for the release of $\mathrm{Ca}^{2+}$ from SR into the cytoplasm via a so-called calcium-induced calcium-release mechanism. Usually, a low heart rate or even a lethal arrhythmia will occur in the mice with an abnormal gene expression of RyR2 [35]. The present data showed that in the model group, the content of RyR2 which is a key SR membrane protein responsible for the $\mathrm{Ca}^{2+}$ release from the SR to cytoplasm increased significantly as compared with the NC group, suggesting that the RyR2 is involved in the cardiac injury described above, while a significant reduction of RyR2 in the EA group in comparison with that in the M group suggests that the SR membrane protein RyR2 participates in the mediation of the aforementioned cardioprotection produced by EA pretreatment.

NCX1 is one of the subtypes of NCX, an antiporter membrane protein mainly responsible for removing $\mathrm{Ca}^{2+}$ from the cytoplasm by exchanging $\mathrm{Na}^{+}$into the cells, so as to participate the balancing of the intracellular $\mathrm{Ca}^{2+}$ concentration. Pathologically, NCX is reported to be associated with the delayed afterdepolarizations which may trigger cardiac arrhythmias [36]. Among the groups in the present study, NCX1 content was not changed significantly. However, a previous study shows that the elevated protein level of NCX1 is downregulated by electroacupuncture pretreatment [37]. The different results may be because the I/R model used in the present study is different from the abovementioned one. In the present study, the reperfusion time is only $15 \mathrm{~min}$, much shorter than $80 \mathrm{~min}$ in the previous study mentioned above. Furthermore, NCX is known to be regulated by $\mathrm{pH}$. Numerous pathological conditions are associated with drops in $\mathrm{pH}$, which in turn affects NCX activity $[38,39]$. Notably, I/R can reduce the intracellular $\mathrm{pH}$, leading to an inhibitory influence on NCX transport that may contribute to the $\mathrm{Ca}^{2+}$ aberrations. Although the NCX1 

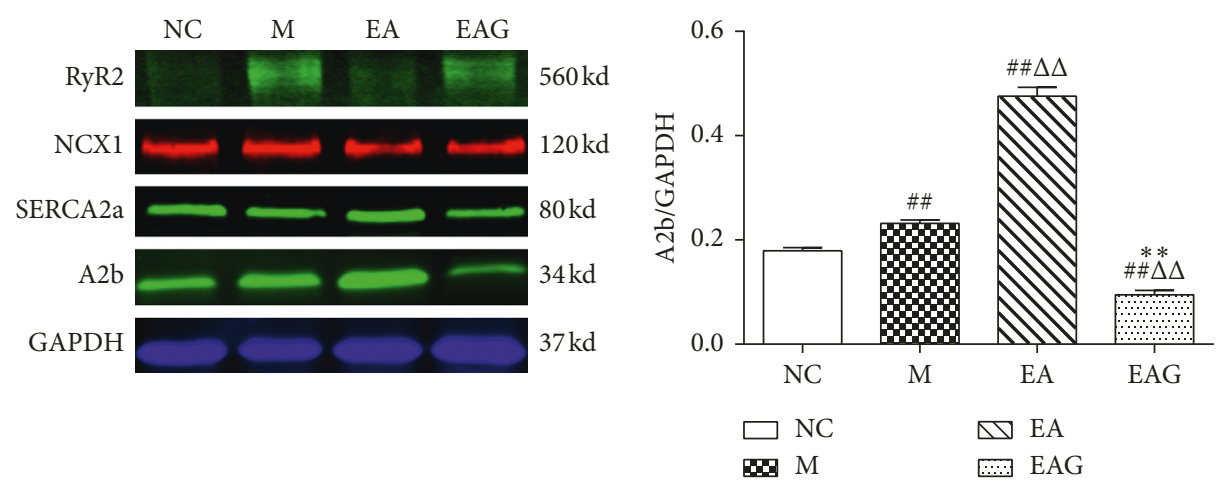

(a)

(b)

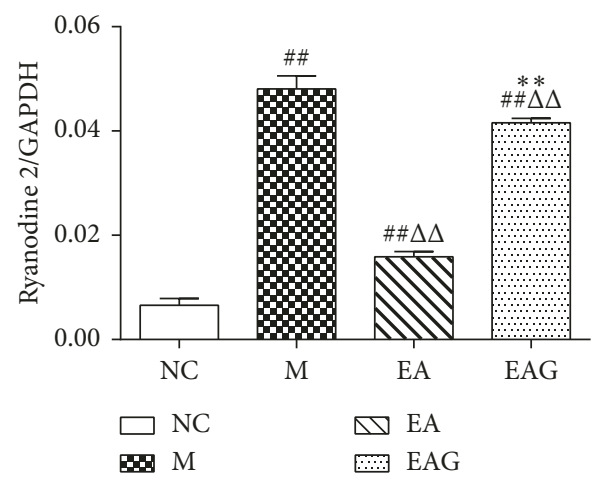

(c)

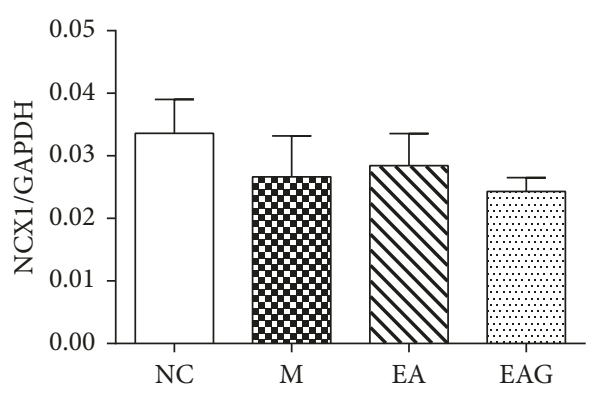

$\begin{array}{ll}\square \mathrm{NC} & \square \mathrm{EA} \\ \mathbf{M} & \square \mathrm{EAG}\end{array}$

(d)

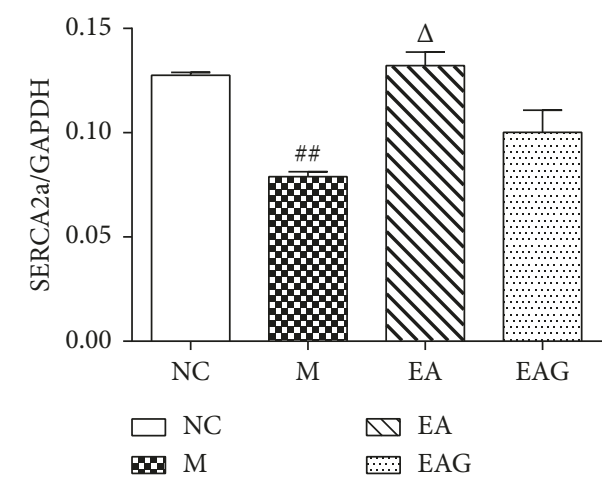

(e)

FIgURE 5: The relative expression of protein in local tissue of injured myocardial tissues. $\left({ }^{\# \#} P<0.01,{ }^{\#} P<0.05\right.$, vs. NC group; ${ }^{\Delta \Delta} P<0.01$, ${ }^{\Delta} P<0.05$, vs. M group; ${ }^{* *} P<0.01,{ }^{*} P<0.05$, vs. EA group, $n=6$ in each group, $\left.\bar{\chi} \pm S E\right)$.

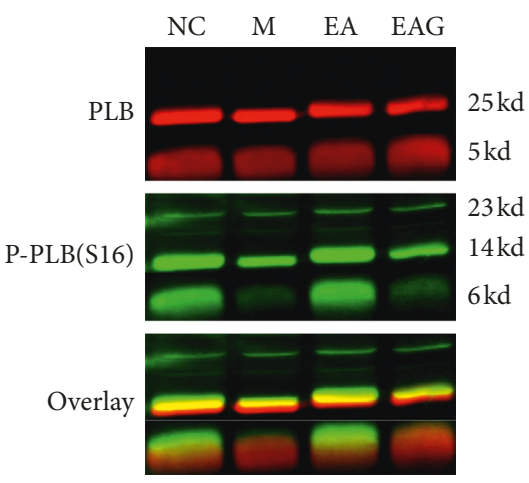

(a)

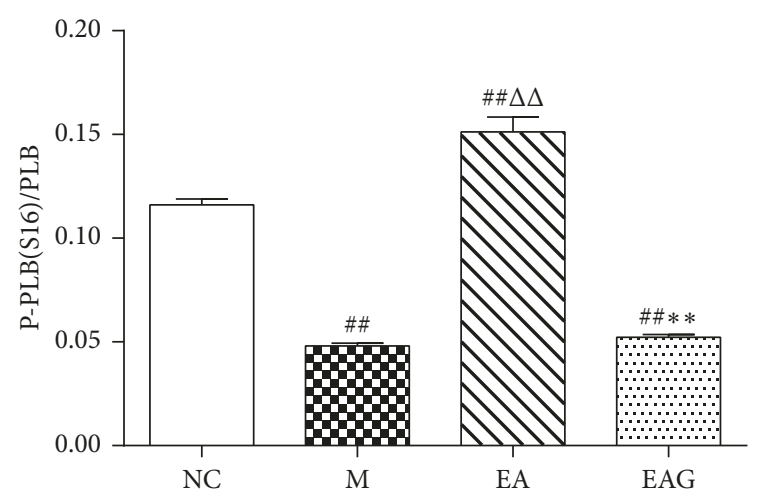

(b)

Figure 6: The ratio of P-PLB/PLB in local tissue of injured myocardial tissues. $\left({ }^{\# \#} P<0.01,{ }^{\#} P<0.05\right.$, vs. NC group; ${ }^{\Delta \Delta} P<0.01,{ }^{\Delta} P<0.05$, vs. M group; ${ }^{* *} P<0.01,{ }^{*} P<0.05$, vs. EA group, $n=6$ in each group, $\left.\bar{\chi} \pm S E\right)$. 


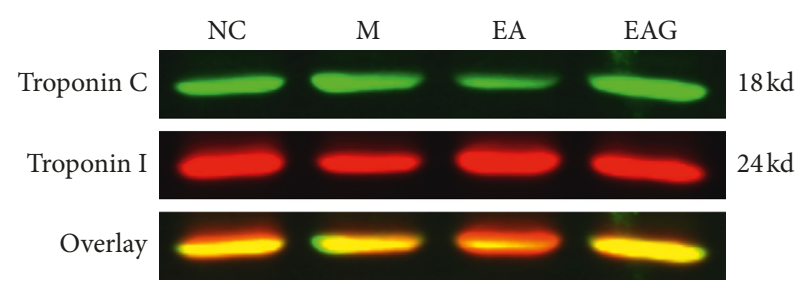

(a)

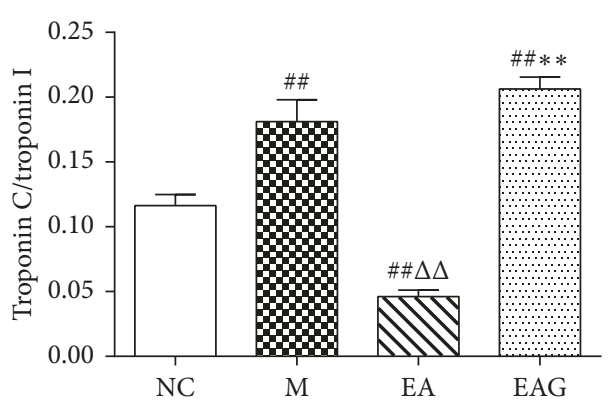

(b)

Figure 7: The ratio of troponin C/troponin I in local tissue of injured myocardial tissues. $\left({ }^{\# \#} P<0.01,{ }^{\#} P<0.05\right.$, vs. NC group; ${ }^{\Delta \Delta} P<0.01$, ${ }^{\Delta} P<0.05$, vs. M group; ${ }^{* *} P<0.01,{ }^{*} P<0.05$, vs. EA group, $n=6$ in each group, $\left.\bar{\chi} \pm S E\right)$.

TABLE 2: The variation of proteins related to the calcium regulation.

\begin{tabular}{|c|c|c|c|}
\hline Receptors/proteins & Roles played by the proteins & EA vs. $M$ & EAG vs. EA \\
\hline $\mathrm{A} 2 \mathrm{~b}$ & Related to calcium regulation & $\uparrow$ & $\downarrow$ \\
\hline Ryanodine 2 & $\begin{array}{l}\text { Releasing } \mathrm{Ca}^{2+} \text { from SR into the cytoplasm and } \\
\text { related to the myocardial contraction }\end{array}$ & $\downarrow$ & $\uparrow$ \\
\hline SERCA $2 \mathrm{a}$ & $\begin{array}{c}\text { Calcium pump to uptake } \mathrm{Ca}^{2+} \text { into SR and related to } \\
\text { myocardial diastolic }\end{array}$ & $\uparrow$ & - \\
\hline NCX1 & $\begin{array}{l}\text { Removing } \mathrm{Ca}^{2+} \text { from the cytoplasm by exchanging } \\
\qquad \mathrm{Na}^{+} \text {into the cells }\end{array}$ & - & - \\
\hline P-PLB(S16)/PLB & $\begin{array}{c}\text { Regulating the function of SERCA2a, related to } \\
\text { uptake intracellular } \mathrm{Ca}^{2+} \text { into SR }\end{array}$ & $\uparrow$ & $\downarrow$ \\
\hline Troponin $\mathrm{C} / \mathrm{I}$ & The binding degree of cardiac myocytes to calcium & $\downarrow$ & $\uparrow$ \\
\hline
\end{tabular}

$\uparrow$, upregulated; $\downarrow$, downregulated; - , unchanged.

content was not changed among the groups, the activity may be changed.

Another important membrane protein related to removal of $\mathrm{Ca}^{2+}$ from the cytoplasm is SERCA2a [40, 41]. During the diastolic period, most intracellular $\mathrm{Ca}^{2+}$ are taken back to the sarcoplasmic reticulum via SERCA2a, known to be prepared for triggering the next myocardial contraction [42]. The enhancement of $\mathrm{Ca}^{2+}$ uptake into the $\mathrm{SR}$ is considered to inhibit the removal of intracellular $\mathrm{Ca}^{2+}$ via NCX [43]. Impaired SERCA2a function is usually implicated in the abnormal diastolic or even systolic dysfunction [44]. As shown in the present results, the content of SERCA2a in the $M$ group was markedly decreased as compared with that in the NC group, indicating that the reduced SERCA2a may at least partially mediate the cardiac dysfunction induced by I/R. In the EA group, the SERCA2a was increased obviously in comparison with the $M$ group, indicating that EA pretreatment could elevate the protein level of SERCA2a in cardiomyocytes, which may contribute to the cardioprotection by enhancing the reuptake of intracellular $\mathrm{Ca}^{2+}$ into SR and decreasing the persistent contraction of cardiomyocytes during the overload of intracellular $\mathrm{Ca}^{2+}$. Actually, in the cardiomyocytes, SERCA is a predominant $\mathrm{Ca}^{2+}$ transporter because of the fact that it can remove more than 10 times intracellular $\mathrm{Ca}^{2+}$ as compared with NCX [45]. Taken together, the results suggest that on the I/R model used in the present study, SERCA2a, not NCX1, plays an important role in the mediation of cardioprotection produced by acupuncture pretreatment.
As an SR membrane protein with 52 amino acids, PLB usually inhibits the calcium-pumping function of SERCA in a reversible way $[46,47]$, mainly inhibiting the uptake of intracellular $\mathrm{Ca}^{2+}$ into the SR performed by SERCA2a. It is well known that heart failure is often accompanied with an impaired function in dealing with $\mathrm{Ca}^{2+}$ in the cardiomyocytes. Studies showed that in the early stage of heart failure, the reduction of phosphorylation of PLB accompanied with or without a decrease of SERCA2a expression led to an increase in the intracellular $\mathrm{Ca}^{2+}$ during diastole, which potentially results in a diastolic dysfunction $[48,49]$. The present study showed that in the EA group, the P-PLB/ $\mathrm{PLB}$ ratio was much higher than that in the $\mathrm{M}$ group. The P-PLB/PLB ratio is helpful for the uptake of intracellular $\mathrm{Ca}^{2+}$ into the $\mathrm{SR}$, so as to possibly improve the impaired cardiac diastolic function.

Contraction of cardiac muscle is driven by an interaction between myosin and actin which is controlled by the transient binding of $\mathrm{Ca}^{2+}$ ions to Cardiac troponin $\mathrm{C}$ (cTn $\mathrm{C}$ ) and activates thin muscle filaments on a beat-to-beat basis [50]. Cardiac troponin I (cTn I) inhibits the actomyosin ATPase on its own in a $\mathrm{Ca}^{2+}$-independent manner, and $\mathrm{Ca}^{2+}$ binding to regulatory sites located in the $\mathrm{NH}_{2}$-terminal domain of cTn $\mathrm{C}$ induces a conformational change that blocks the inhibitory action of cTn I and triggers muscle contraction [51, 52]. CTn I also plays a central role in ischemia-systolic dysfunction. Also, it is an inhibitory subunit of the troponin complex which acts as a $\mathrm{Ca}^{2+}$-dependent molecular switch by shuttling between actin (diastolic) and 
troponin $\mathrm{C}$ (systolic) [53]. When $\mathrm{Ca}^{2+}$ level is low, there is little interaction between cTn C and cTn I. It is a result that cTn I interacts with actin and inhibits it. When cTn $\mathrm{C}$ interacts with $\mathrm{Ca}^{2+}$, the interaction between $\mathrm{cTn} C$ and cTn I is enhanced to alleviate the interaction between cTn I and actin. $\mathrm{Ca}^{2+}$ binding to low-affinity sites in the N-terminal region of cTn $\mathrm{C}$ is the first step in the sequence of events associated with activation of myocardial contractile proteins [54]. CTn I regulates $\mathrm{Ca}^{2+}$ sensitivity on myofilaments by phosphorylation and intracellular environment and regulates myocardial contractility in a specific isomerism [55]. Calcium overload in cardiac myocytes is one of the important causes of myocardial ischemia injury [56]. Our previous experiments have also confirmed that electroacupuncture pretreatment can reduce the openness of L-type $\mathrm{Ca}^{2+}$ channels in cardiac myocytes that mimic whole-heart ischemia and ultimately alleviate myocardial injury caused by intracardiac calcium overload caused by ischemia [57]. The results show that $\mathrm{cTn} \mathrm{C} / \mathrm{I}$ ratio in the model group was significantly increased, which indicates that the cardiomyocytes were easier to bond with $\mathrm{Ca}^{2+}$, and the cardiomyocytes were prone to calcium overload injury, while the cTn C/I ratio in the EA group was significantly decreased, showing that the binding of cardiac myocytes to calcium was decreased and the occurrence of calcium overload after myocardial cell injury was reduced.

\section{Conclusions}

In conclusion, electroacupuncture pretreatment could reduce ischemia and reperfusion-induced myocardial injury via increasing possibly the $\mathrm{A} 2 \mathrm{~b}$ content and regulating the key $\mathrm{Ca}^{2+}$ signaling components, namely inhibiting RyR2 and enhancing P-PLB(S16)/PLB ratio and SERCA2a proteins, so as to diminish the intracellular $\mathrm{Ca}^{2+}$ overload and consequently lessen the myocardial injury.

\section{Data Availability}

The data used to support the findings of this study are available from the corresponding author upon request.

\section{Conflicts of Interest}

The authors declare that there are no conflicts of interest regarding the publication of this paper.

\section{Authors' Contributions}

The experiments were done by Qiu-Fu Dai. Juan-Juan Xin, Qun Liu, and Xiang-Hong Jing provided advice on the statistical analyses and data interpretation. Qiu-Fu Dai, Xiao-Chun Yu, and Jun-Hong Gao drafted and finalized the paper. Xiao-Chun $\mathrm{Yu}$ was responsible for the conception, design, and supervision of the implementation of the study.

\section{Acknowledgments}

This work was supported by the National Natural Science Foundation of China (nos. 81774439 and 81674089).

\section{References}

[1] Committee of Exports on Rational Drug Use, National Health and Family Planning Commission of the P.R. China and Chinese Pharmacists Association, Guidelines for Rational Use of Coronary Heart Disease, China Academic Journal Electronic Publishiing House, Minneapolis, MN, USA, 2nd edition, 2018.

[2] H. K. Eltzschig and T. Eckle, "Ischemia and reperfusion-from mechanism to translation," Nature Medicine, vol. 17, no. 11, pp. 1391-1401, 2011.

[3] A. L. Moens, M. J. Claeys, J. P. Timmermans, and C. J. Vrints, "Myocardial ischemia/reperfusion-injury, a clinical view on a complex pathophysiological process," International Journal of Cardiology, vol. 100, no. 2, pp. 179-190, 2005.

[4] S. W. Ely, R. M. Jr Mentzer, R. D. Lasley et al., "Functional and metabolic evidence of enhanced myocardial tolerance to ischemia and reperfusion with adenosine," Journal of Thoracic and Cardiovascular Surgery, vol. 90, no. 4, pp. 549-556, 1985.

[5] R. Schulz and M. V. Cohen, "Signal transduction of ischemic preconditioning," Cardiovascular Research, vol. 52, no. 2, pp. 181-198, 2001.

[6] G. F. Baxter, M. S. Marber, V. C. Patel, and D. M. Yellon, "Adenosine receptor involvement in a delayed phase of myocardial protection 24 hours after ischemic preconditioning," Circulation, vol. 90, no. 6, pp. 2993-3000, 1994.

[7] Z.-Q. Zhao, J. M. Budde, C. Morris et al., "Adenosine attenuates reperfusion-induced apoptotic cell death by modulating expression of Bcl-2 and Bax proteins," Journal of Molecular and Cellular Cardiology, vol. 33, no. 1, pp. 57-68, 2001.

[8] J. Gile and T. Eckle, "ADORA2b signaling in cardioprotection," Journal of Nature and Science, vol. 2, no. 10, 2016.

[9] T. Eckle, T. Krahn, A. Grenz et al., "Cardioprotection by ecto$5^{\prime}$-nucleotidase (CD73) and A 2B adenosine receptors," Circulation, vol. 115, no. 12, pp. 1581-1590, 2007.

[10] Q. Wang, D. Liang, F. Wang et al., "Efficacy of electroacupuncture pretreatment for myocardial injury in patients undergoing percutaneous coronary intervention: a randomized clinical trial with a 2-year follow-up," International Journal of Cardiology, vol. 194, pp. 28-35, 2015.

[11] L. Yang, J. Yang, Q. Wang et al., "Cardioprotective effects of electroacupuncture pretreatment on patients undergoing heart valve replacement surgery: a randomized controlled trial," The Annals of Thoracic Surgery, vol. 89, no. 3, pp. 781-786, 2010.

[12] P. K. Mehta, D. M. Polk, X. Zhang et al., “A randomized controlled trial of acupuncture in stable ischemic heart disease patients," International Journal of Cardiology, vol. 176, no. 2, pp. 367-374, 2014.

[13] J. C. Longhurst, "Electroacupuncture treatment of arrhythmias in myocardial ischemia," American Journal of Physiology-Heart and Circulatory Physiology, vol. 292, no. 5, pp. H2032-H2034, 2007.

[14] A. Richter, J. Herlitz, and A. Hjalmarson, "Effect of acupuncture in patients with angina pectoris," European Heart Journal, vol. 12, no. 2, pp. 175-178, 1991.

[15] T. Takano, X. Chen, F. Luo et al., "Traditional acupuncture triggers a local increase in adenosine in human subjects," The Journal of Pain, vol. 13, no. 12, pp. 1215-1223, 2012.

[16] Y. Zhuang, "The study on characteristics of purinergic signal change on the involved meridian and the protective effect of acupuncture at selected acupoint on the pericardium meridian 
for myocardial ischemia rat via cardiac $A_{2 b}$ receptor," Doctoral thesis, Cheng Du University of Traditional Chinese Medicine, Chengdu, China, 2015.

[17] M. Mirabet, J. Mallol, C. Lluis, and R. Franco, "Calcium mobilization in Jurkat cells via A2badenosine receptors," British Journal of Pharmacology, vol. 122, no. 6, pp. 10751082, 1997.

[18] Z. Gao, T. Chen, M. J. Weber, and J. Linden, “A2B adenosine and P2Y2 receptors stimulate mitogen-activated protein kinase in human embryonic kidney-293 cells," Journal of Biological Chemistry, vol. 274, no. 9, pp. 5972-5980, 1999.

[19] M. Panjehpour, M. N. Castro, and K. N. Klotz, "Human breast cancer cell line MDA-MB-231 expresses endogenous A2B adenosine receptors mediating a $\mathrm{Ca}^{2+}$ signal," British Journal of Pharmacology, vol. 145, no. 2, pp. 211-218, 2005.

[20] C.-X. Sun, H. Zhong, A. Mohsenin et al., "Role of A2B adenosine receptor signaling in adenosine-dependent pulmonary inflammation and injury," Journal of Clinical Investigation, vol. 116, no. 8, pp. 2173-2182, 2006.

[21] Q. F. Dai, S. Y. Wu, J. J. Xin et al., "Effects of electroacupuncture pretreatment on modified acute myocardial ischemia reperfusion model rats," China Journal of Traditional Chinese Medicine and Pharmacy, vol. 34, no. 5, pp. 2002-2006, 2019.

[22] A. Azhar and H. M. El-Bassossy, "Pentoxifylline alleviates cardiac ischemia and dysfunction following experimental angina in insulin resistance," PLoS One, vol. 9, no. 5, Article ID e98281, 2014.

[23] T. Wong, A. Y. Lee, and K. K. Tai, "Effects of drugs interacting with opioid receptors during normal perfusion or ischemia and reperfusion in the isolated rat heart- an attempt to identify cardiac opioid receptor subtype(s) involved in arrhythmogenesis," Journal of Molecular and Cellular Cardiology, vol. 22, no. 10, pp. 1167-1175, 1990.

[24] M. J. Curtis and M. J. A. Walker, "Quantification of arrhythmias using scoring systems: an examination of seven scores in an in vivo model of regional myocardial ischaemia," Cardiovascular Research, vol. 22, no. 9, pp. 656-665, 1988.

[25] L. H. Li, W. H. Chen, and H. Zheng, "An optimized modification of TTC staining method for better detection of myocardial ischemia-reperfusion injury in rats," Acta Laboratorium Animalis Scientia Sinica, vol. 5, pp. 75-78, 2014.

[26] D. Wang, R. Zhang, X. Zhou et al., "Electroacupuncture pretreatment ameliorates myocardial ischaemia/reperfusion injury through regulation of cannabinoid receptor type 2," European Heart Journal, vol. 17, 2015.

[27] Q. Zeng, H. He, X.-B. Wang et al., "Electroacupuncture preconditioning improves myocardial infarction injury via enhancing AMPK-dependent autophagy in rats," BioMed Research International, vol. 2018, Article ID 1238175, 2018.

[28] H. K. Eltzschig, S. K. Bonney, and T. Eckle, "Attenuating myocardial ischemia by targeting A2B adenosine receptors," Trends in Molecular Medicine, vol. 19, no. 6, pp. 345-354, 2013.

[29] I. Feoktistov, A. E. Goldstein, S. Ryzhov et al., "Differential expression of adenosine receptors in human endothelial cells," Circulation Research, vol. 90, no. 5, pp. 531-538, 2002.

[30] I. Feoktistov, S. Ryzhov, A. E. Goldstein, and I. Biaggioni, "Mast cell-mediated stimulation of angiogenesis," Circulation Research, vol. 92, no. 5, pp. 485-492, 2003.

[31] N. Goldman, M. Chen, T. Fujita et al., "Adenosine A1 receptors mediate local anti-nociceptive effects of acupuncture," Nature Neuroscience, vol. 13, no. 7, pp. 883-888, 2010.

[32] S. F. Lu, Y. X. Tang, Y. J. Ding et al., "Effects of electroacupuncture on the expression of adenosine receptors in the heart tissue of myocardial ischemia rats," Zhongguo Zhen Jiu = Chinese Acupuncture and Moxibustion, vol. 38, no. 2, pp. 173-179, 2018.

[33] K. Pittas, D. A. Vrachatis, C. Angelidis et al., "The role of calcium handling mechanisms in reperfusion injury," Current Pharmaceutical Design, vol. 24, no. 34, pp. 4077-4089, 2018.

[34] D. A. Eisner, J. L. Caldwell, K. Kistamás, and A. W. Trafford, "Calcium and excitation-contraction coupling in the heart," Circulation Research, vol. 121, no. 2, pp. 181-195, 2017.

[35] M. J. Bround, P. Asghari, R. B. Wambolt et al., "Cardiac ryanodine receptors control heart rate and rhythmicity in adult mice," Cardiovascular Research, vol. 96, no. 3, pp. 372-380, 2012.

[36] Z. A. Nagy, V. László, T. András et al., "Selective inhibition of sodium-calcium exchanger by SEA-0400 decreases early and delayed after depolarization in canine heart," British Journal of Pharmacology, vol. 143, no. 7, pp. 827-831, 2010.

[37] P. Liu, Y. Tian, and X. U. Shiyuan, "Mediated protective effect of electroacupuncture pretreatment by miR-214 on myocardial ischemia/reperfusion injury," Journal of Geriatric Cardiology, vol. 11, no. 4, pp. 303-310, 2014.

[38] S. John, B. Kim, R. Olcese, J. I. Goldhaber, and M. Ottolia, "Molecular determinants of $\mathrm{pH}$ regulation in the cardiac $\mathrm{Na}^{+}$$\mathrm{Ca}^{2+}$ exchanger," The Journal of General Physiology, vol. 150, no. 2, pp. 245-257, 2018.

[39] C. D. Garciarena, J. B. Youm, P. Swietach, and R. D. VaughanJones, " $\mathrm{H}^{+}$-activated $\mathrm{Na}^{+}$influx in the ventricular myocyte couples $\mathrm{Ca}^{2+}$-signalling to intracellular $\mathrm{pH}$," Journal of Molecular and Cellular Cardiology, vol. 61, pp. 51-59, 2013.

[40] L. M. Crespo, C. J. Grantham, and M. B. Cannell, "Kinetics, stoichiometry and role of the $\mathrm{Na}-\mathrm{Ca}$ exchange mechanism in isolated cardiac myocytes," Nature, vol. 345, no. 6276, pp. 618-621, 1990.

[41] N. Negretti, S. C. O’Neill, and D. A. Eisner, “The relative contributions of different intracellular and sarcolemmal systems to relaxation in rat ventricular myocytes," Cardiovascular Research, vol. 27, no. 10, pp. 1826-1830, 1993.

[42] W. Luo, B. M. Wolska, I. L. Grupp et al., "Phospholamban gene dosage effects in the mammalian heart," Circulation Research, vol. 78, no. 5, pp. 839-847, 1996.

[43] H. Satoh, K. S. Ginsburg, K. Qing, H. Terada, H. Hayashi, and D. M. Bers, "KB-R7943 block of $\mathrm{Ca}^{2+}$ influx via $\mathrm{Na}^{+} / \mathrm{Ca}^{2+}$ exchange does not alter twitches or glycoside inotropy but prevents $\mathrm{Ca}^{2+}$ overload in rat ventricular myocytes," Circulation, vol. 101, no. 12, pp. 1441-1446, 2000.

[44] M. A. H. Talukder, A. Kalyanasundaram, L. Zuo et al., "Is reduced SERCA2a expression detrimental or beneficial to postischemic cardiac function and injury? Evidence from heterozygous SERCA2a knockout mice," American Journal of Physiology-Heart and Circulatory Physiology, vol. 294, no. 3, pp. H1426-H1434, 2008.

[45] J. W. Bassani, R. A. Bassani, and D. M. Bers, "Relaxation in rabbit and rat cardiac cells: species-dependent differences in cellular mechanisms," The Journal of Physiology, vol. 476, no. 2, pp. 279-293, 1994.

[46] M. Tada, M. A. Kirchberger, D. I. Repke et al., "The stimulation of calcium transport in cardiac sarcoplasmic reticulum by adenosine $3^{\prime}: 5^{\prime}$-monophosphate-dependent protein kinase," Journal of Biological Chemistry, vol. 249, no. 19, pp. 6174-6180, 1974.

[47] D. M. Bers, "Cardiac excitation-contraction coupling," $\mathrm{Na}$ ture, vol. 415, no. 6868, pp. 198-205, 2002.

[48] A. R. Marks, "Calcium cycling proteins and heart failure: mechanisms and therapeutics," Journal of Clinical Investigation, vol. 123, no. 1, pp. 46-52, 2013. 
[49] E. G. Kranias and R. J. Hajjar, "Modulation of cardiac contractility by the phopholamban/SERCA2a regulatome," Circulation Research, vol. 110, no. 12, pp. 1646-1660, 2012.

[50] T. Kobayashi and R. J. Solaro, "Calcium, thin filaments, and the integrative biology of cardiac contractility," Annual Review of Physiology, vol. 67, no. 1, pp. 39-67, 2005.

[51] C. S. Farah, C. A. Miyamoto, C. H. Ramos et al., "Structural and regulatory functions of the $\mathrm{NH} 2$ - and $\mathrm{COOH}$-terminal regions of skeletal muscle troponin I," Journal of Biological Chemistry, vol. 269, no. 7, pp. 5230-5240, 1994.

[52] C. S. Farah and F. C. Reinach, "The troponin complex and regulation of muscle contraction," The FASEB Journal, vol. 9, no. 9, pp. 755-767, 1995.

[53] J. M. Metzger and M. V. Westfall, "Covalent and noncovalent modification of thin filament action," Circulation Research, vol. 94, no. 2, pp. 146-158, 2004.

[54] J. A. Putkey, H. L. Sweeney, and S. T. Campbell, "Site-directed mutation of the trigger calcium-binding sites in cardiac troponin C," Journal of Biological Chemistry, vol. 264, no. 21, pp. 12370-12378, 1989.

[55] R. J. Solaro, J. A. Lee, J. C. Kentish, and D. G. Allen, "Effects of acidosis on ventricular muscle from adult and neonatal rats," Circulation Research, vol. 63, no. 4, pp. 779-787, 1988.

[56] H. Zhou, J. Wang, P. Zhu, S. Hu, and J. Ren, "Ripk3 regulates cardiac microvascular reperfusion injury: the role of IP3Rdependent calcium overload, XO-mediated oxidative stress and F-action/filopodia-based cellular migration," Cellular Signalling, vol. 45, pp. 12-22, 2018.

[57] J. H. Gao, "Pretreatment of electroacupuncture attenuates myocardial injury induced by ischemia and reperfusion in the rats-involvement of $\beta 1$-adrenergic receptor signaling pathway," Doctoral thesis, China Academy of Chinese Medical Science, Beijing, China, 2006. 


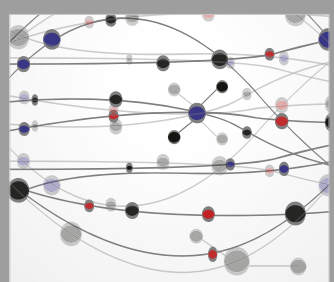

The Scientific World Journal
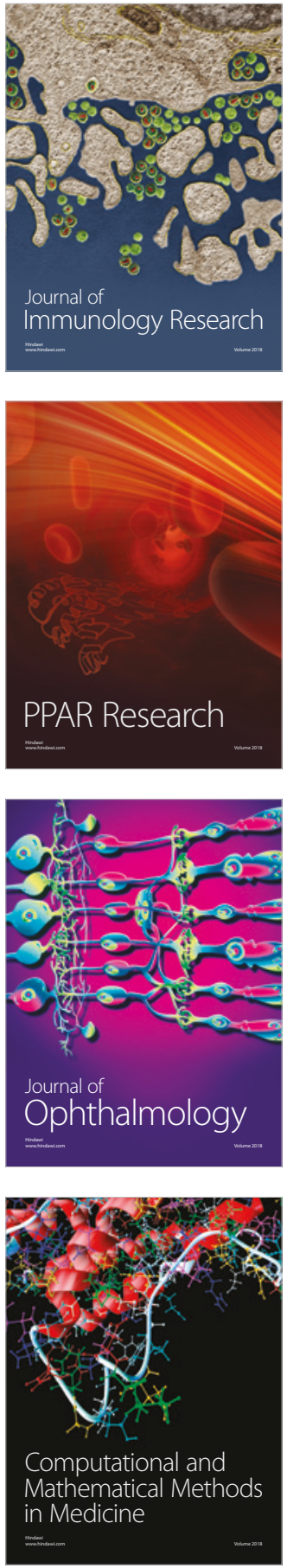

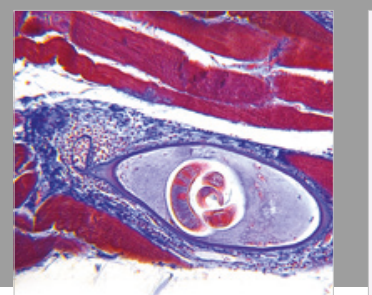

Gastroenterology Research and Practice

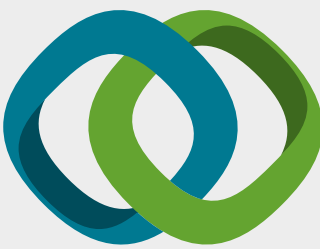

\section{Hindawi}

Submit your manuscripts at

www.hindawi.com
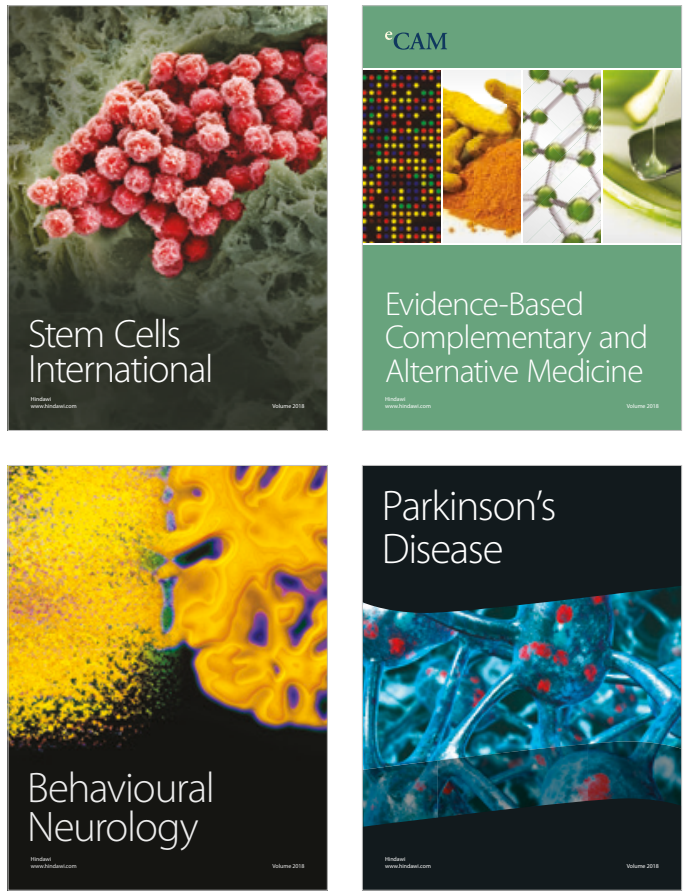

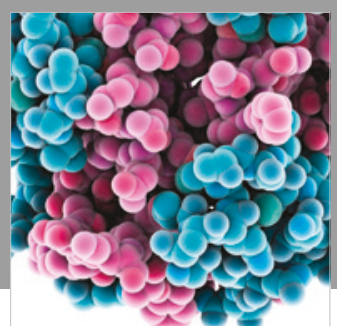

ournal of

Diabetes Research

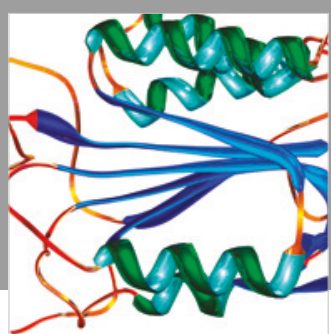

Disease Markers
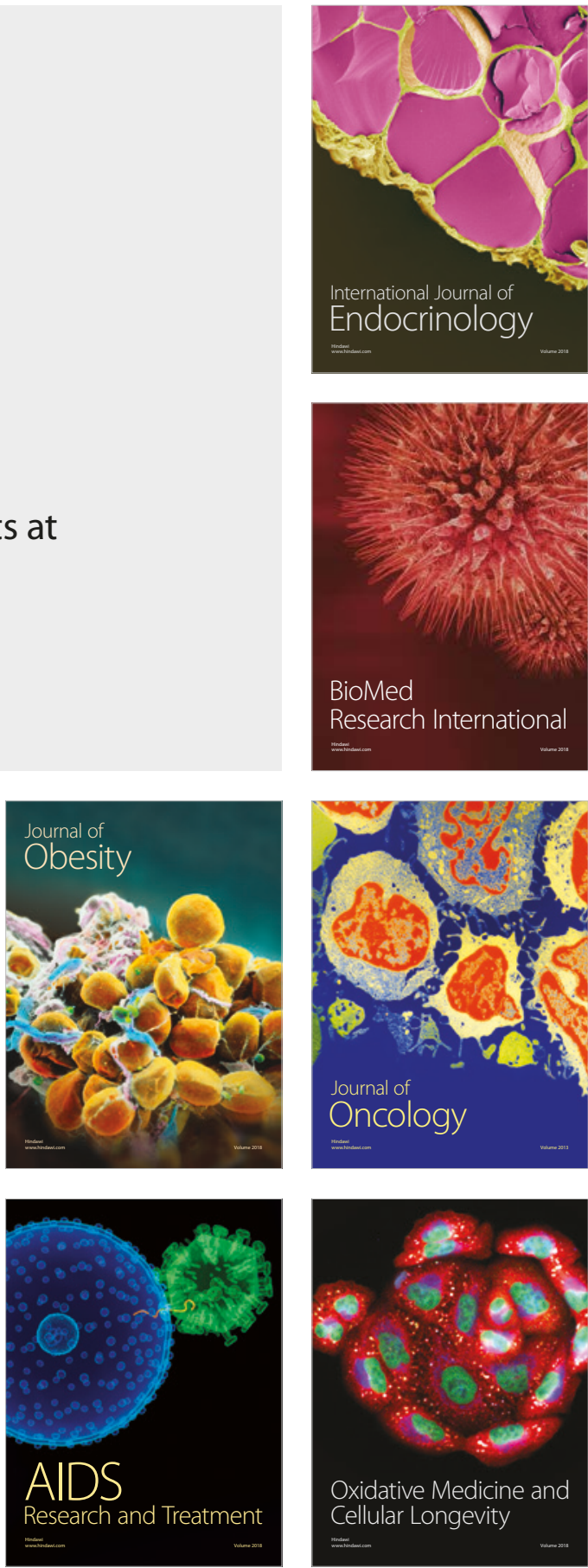